\title{
ACME: an Affinity-based Cas9 Mediated Enrichment method for targeted nanopore sequencing.
}

\author{
Shruti V Iyer ${ }^{1,2}$, Melissa Kramer ${ }^{1}$, Sara Goodwin ${ }^{1}$, W. Richard McCombie ${ }^{1}$ \\ 1. Genome Center, Cold Spring Harbor Laboratory, Cold Spring Harbor, NY \\ 2. Program in Genetics, Stony Brook University, Stony Brook, NY
}

\begin{abstract}
:
Targeted sequencing significantly improves accuracy and coverage and aids in providing the depth necessary to detect rare alleles in a heterogenous population of cells. Until the introduction of nanopore Cas9 Targeted-Sequencing (nCATS), a lack of efficient long-read compatible targeting techniques made it difficult to study specific regions of interest on longread platforms. Existing nCATS-based strategies are currently limited by the per molecule target lengths capturable $(<30 \mathrm{~kb})$, requiring several Cas9 guides to tile across larger regions of interest, ultimately reducing the number of targets that can be surveyed per reaction. Also, longer read lengths help reduce mapping errors, making it more likely that complex structural rearrangements can be resolved. Absence of a background reduction step in nCATS also increases the competition between non-target and target fragments in the sequencing pool for pore occupancy, decreasing the overall percentage of on-target reads. To address this, we

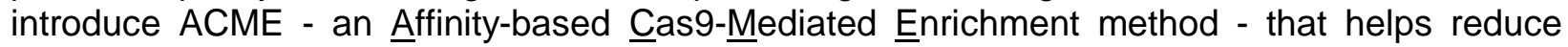
background reads, increasing on-target coverage and size of target regions that can be spanned with single reads to $100 \mathrm{~kb}$.
\end{abstract}

ACME uses a HisTag-based isolation and pulldown of Cas-9 bound non-target reads, reducing the background noise in sequencing. We designed a panel of guide RNAs targeting 10 genes to enrich for specific regions of the cancer genome and tested them in two breast cell lines - MCF $10 \mathrm{~A}$ and SK-BR-3. These gene targets spanned different size ranges (10kb to $150 \mathrm{~kb}$ ) allowing us to identify the largest target sizes that could be optimally captured by single molecules spanning the entire region. When compared with using just nCATS, the ACME method for background reduction increased the overall coverage across the entire length of all targets by 2fold to 25-fold. By using ACME to eliminate smaller competing non-targets from the sequencing library, we saw a 3- to 7 -fold increase in the number of reads spanning $100 \%$ of the gene targets when compared to nCATS. For one of our larger targets, BRCA2, we observed $>60$-fold target enrichment, close to $70 x$ coverage, and 3-20 reads spanning the entire $95 \mathrm{~kb}$ target. We observed an increase in enrichment, depth, and number of whole gene spanning reads for other genes on the panel as well across both cell lines, with enrichment as high as 4000-fold for some genes. Furthermore, ACME identified all SVs previously called within our targets by ONT and PacBio whole genome sequencing and performed on par with these platforms for SNP detection when compared with Illumina short-read whole genome sequencing.

\section{Introduction:}

Cancer genomes have largely been evaluated using short-read sequencing technologies, which lack sensitivity and exhibit very high false positive rates (up to 89\%) in SV detection ${ }^{1-4}$. Longread sequencing methods such as single-molecule real-time sequencing (SMRT) ${ }^{5}$ and nanopore sequencing ${ }^{6}$ have the ability to detect SVs with $>=95 \%$ sensitivity and specificity ${ }^{7-9}$. However, the difficulty in generating high enough sequence depth to detect rare alleles in a heterogeneous cell mix is even more difficult than with short reads because of the higher sequencing cost associated with long-read technologies. Targeted sequencing dramatically 
changes our ability to study the genome by facilitating higher sample throughput than whole genome sequencing and improves accuracy by increasing the read depth coverage ${ }^{10}$. With targeted long-read sequencing, the goal is to be able to cover an entire target with several single contiguous reads spanning the whole region. Reads covering whole target regions minimize mapping errors due to SVs, aiding in their detection.

A Cas9-based enrichment approach called nanopore Cas9 Targeted-Sequencing (nCATS) was adapted for the Oxford Nanopore Technologies (ONT) platform by Gilpatrick et al., with singleended cleavage variations developed by Stangl et al. ${ }^{11}$ to detect fusion events and Watson et al. ${ }^{12}$ to detect genomic duplications. While nCATS is a fast and effective long-read targeting approach, its two major limitations are: 1$)$ Relatively shorter read lengths ( $<30 \mathrm{~kb}$ ) leading to few or no reads that span larger targets from end to end. This would require several crRNA guides to tile through larger target regions, thereby limiting the number of targets that can be investigated in a single run. 2) Lack of an efficient background reduction step. The nCATS sequencing pool consists of target DNA fragments along with Cas9-bound and non-Cas9 bound dephosphorylated fragments. Higher ratio of non-target to target DNA in the prepared library often results in shorter non-target fragments competing with long targets for pore occupancy, effectively reducing total target depth.

Two groups have adapted the principle behind nCATS to perform exonuclease digestion of nontarget DNA. Cas9-based Background Elimination (CaBagE) ${ }^{13}$ and Negative Enrichment ${ }^{14}$ focus on protecting target DNA ends with Cas9, followed by exonuclease digestion of non-target reads. However, both methods have only achieved target read lengths of $<35 \mathrm{~kb}$. It is also important to note that this approach poses an additional risk of digesting target fragments that may have breaks between the Cas9 binding sites and are not protected by Cas9. Despite its potential, this approach failed to outperform nCATS in its yield and target coverage further highlighting the need for an efficient background reduction step with Cas9-mediated targeting. More recently, computational target enrichment strategies known as adaptive sequencing have been introduced through ReadFish ${ }^{15}$ and UNCALLED ${ }^{16}$, where non-target reads are "rejected", allowing only target sequences to pass through the pores and be sequenced fully. However, these approaches required the DNA to be sheared to $8-15 \mathrm{~kb}$, impacting the ability to detect larger variants.

To address these limitations, we made modifications to the nCATS protocol to facilitate longer target capture and sequencing by reducing background fragments. We developed an Affinity-

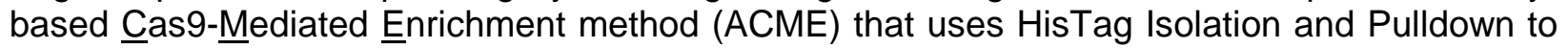
remove background DNA. nCATS uses High-Fidelity (HiFi) Cas9 Nuclease that contains a 6Histidine tag at its C-terminal. After the Cas9 cleavage and dA tailing step in nCATS, the Cas9 enzyme remains bound to the PAM-distal end i.e., non-target DNA side. Cas 9 binding affinity is stronger at the PAM-distal end than the PAM-proximal side of the cleavage site ${ }^{17}$, thereby protecting the non-target DNA end from subsequent sequencing adapter ligation. By introducing His-Tag specific magnetic beads at this step, we were able to pull down Cas- 9 bound non-target fragments from the sample, allowing more target DNA to make it onto the flowcells (Fig. 1). We designed a size titration panel consisting of important cancer genes of different size ranges $10,20,40,80,150 \mathrm{~kb}$ - to identify the largest possible target that could be effectively captured and sequenced as contiguous single fragments using ACME. We used ACME on this panel in two breast cell lines - MCF $10 \mathrm{~A}^{18}$ and SK-BR-3 ${ }^{19}$ and compared its performance with nCATS for Cas9 targeting in MCF 10A and with PacBio and ONT whole genome sequencing in SK-BR3 to detect SVs. 


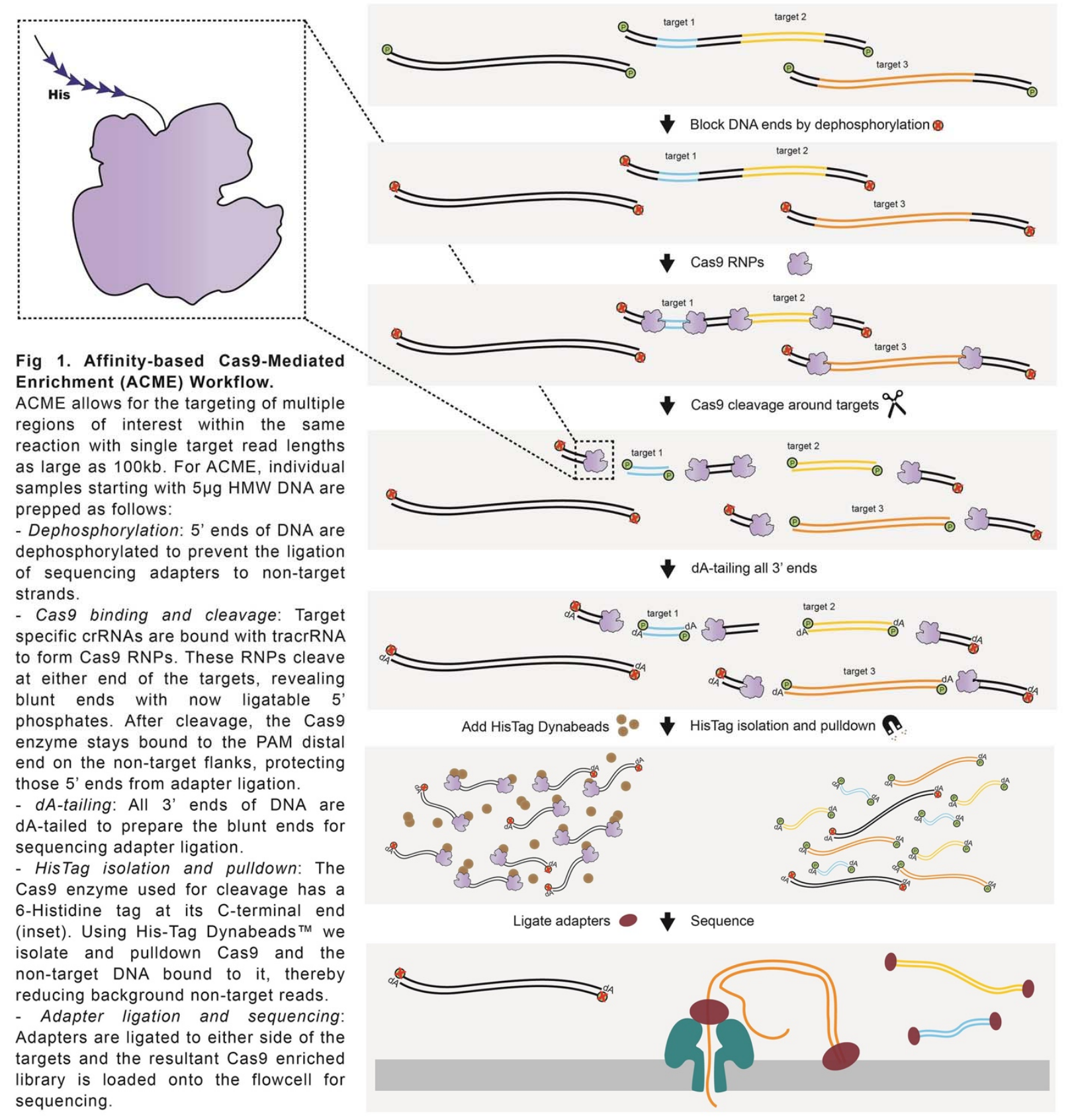

\section{Results:}

Targeting a 200kb region around BRCA1

For our first pass of nCATS we used guides designed by Sage Sciences that targeted a 200kb region around the BRCA1 gene ${ }^{20}$ in SK-BR-3 to test the upper limits of potential target sizes using this method. This run generated a single $198 \mathrm{~kb}$ read spanning the entire BRCA1 region and somewhat higher on-target coverage (Fig. 2A). This library was made from a single sample prep starting with $5 \mu \mathrm{g}$ of HMW DNA. We also replicated these efforts in MCF 10A (Table 1, Supplemental Fig. 1A), a normal breast cell line. To improve on-target reads (Table 1), we modified nCATS to incorporate higher DNA inputs and to reduce background/non-target reads. 
bioRxiv preprint doi: https://doi.org/10.1101/2022.02.03.478550; this version posted February 5, 2022. The copyright holder for this preprint (which was not certified by peer review) is the author/funder, who has granted bioRxiv a license to display the preprint in perpetuity. It is made available under aCC-BY-NC-ND 4.0 International license.

Table 1. Run summaries for the different BRCA1 Cas9-mediated targeted sequencing runs. Enrichment is relative to expected genome abundance for the total observed yield.

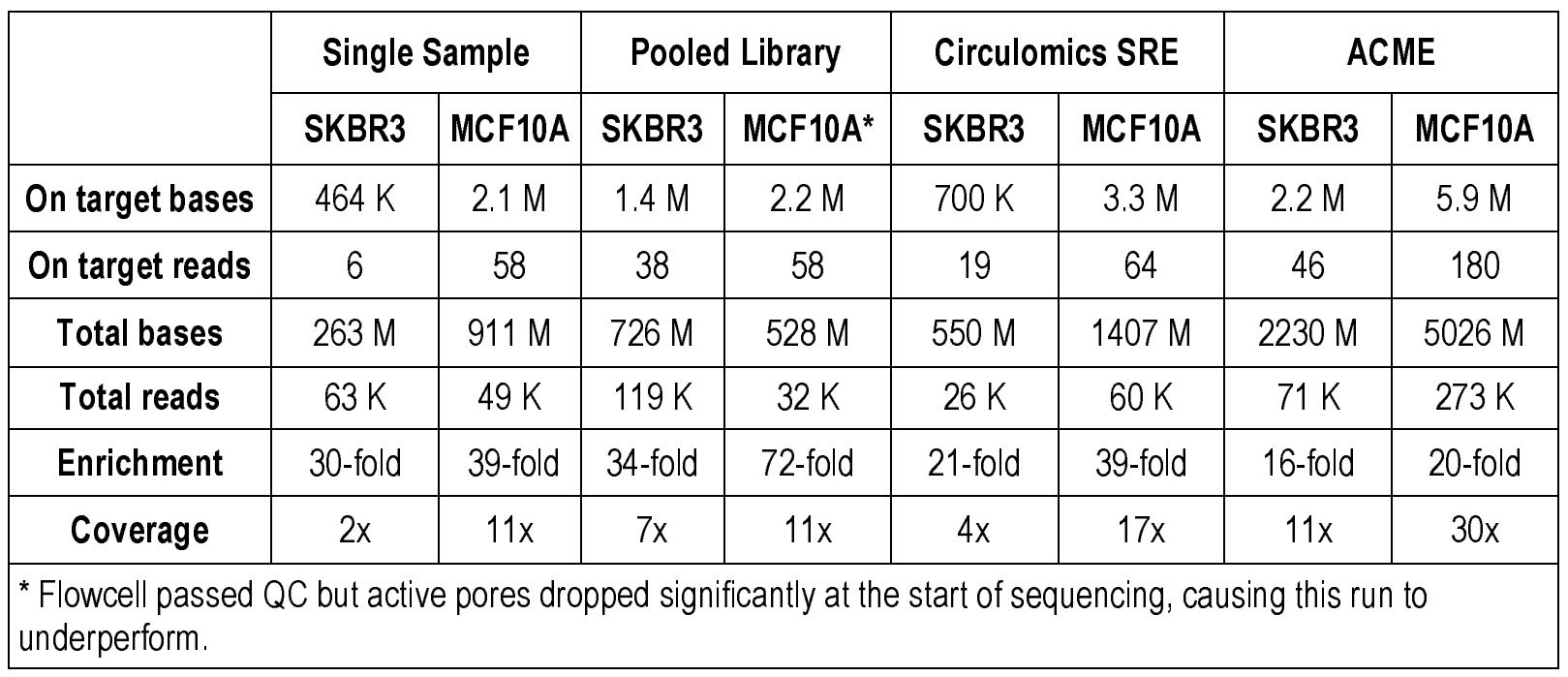

We tried three different modifications to improve depth across large targets of interest as described below:

\section{Pooled Library}

Preparation: Given that we were targeting a single large region and nCATS involves no target

amplification, four identical sample preps were pooled together to ensure sufficient copies of our target made their way to the sequencing pool. This prep gave us better target enrichment with $35-60$ reads on target and a 35-70fold enrichment (Table 1). However, the on-target reads were not long enough to include the entire target region, with drops in coverage around the center of the

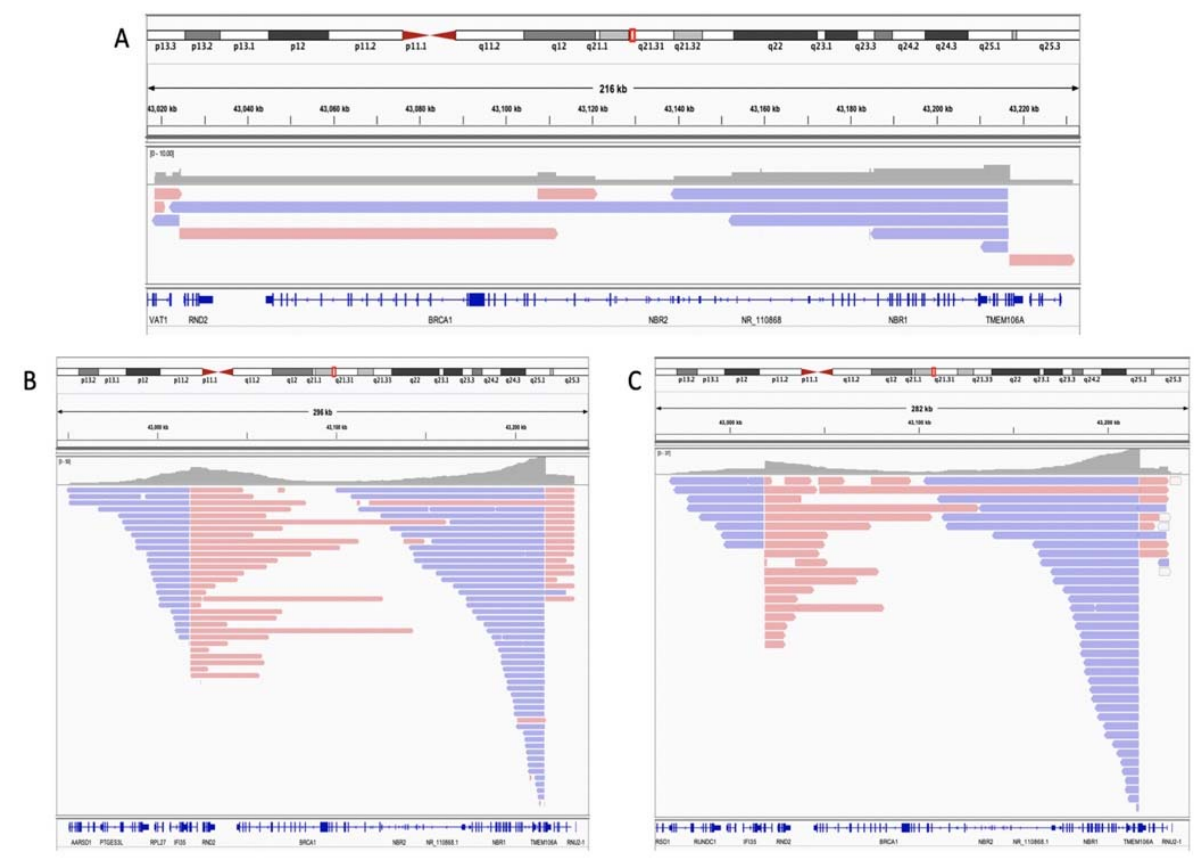

Fig. 2 IGV plots representing reads that mapped to the $B R C A 1$ gene in different Cas9-mediated targeting libraries prepared from MCF 10A \& SK-BR-3 DNA. Plus strand reads are shown in pink and minus strand reads in blue. A: Single sample library prep using SK-BR-3 DNA (nCATS). B: Pooled library prepared using 4 identical preps of MCF 10A DNA with the incorporation of Circulomics Short Read Eliminator kit (SRE). C: Pooled library prepared using 4 identical preps of SK-BR3 DNA with the incorporation of ACME. 
region of interest (Supplemental Fig. 1B).

Circulomics Short Read Elimination (SRE): We used the Short Read Eliminator kit from Circulomics Inc. to reduce potentially competing background reads $<25 \mathrm{~kb}$ in length and boost the coverage of our longer BRCA1 fragments. While this modification did not work well for SKBR-3 with only 19 reads on target (Table 1, Supplemental Fig. 1C), the MCF 10A performed much better with one $142 \mathrm{~kb}$ read and 64 reads on target (Table 1, Fig. 2B).

Affinity-based Cas9-Mediated Enrichment (ACME): The Alt-R® S.p. HiFi Cas9 Nuclease from Integrated DNA Technologies (IDTß) used in the ONT Cas9 protocol contains a C-terminal 6His tag. Additionally, the Cas9 remains bound to the PAM-distal side (non-target DNA) after cleavage, protecting it from sequencing adapter ligation. By using Invitrogen ${ }^{\mathrm{TM}}$ Dynabeads ${ }^{\mathrm{TM}}$ His-Tag Isolation and Pulldown, we selected against the background by enriching for the nonCas9 bound regions of interest (Fig. 1). This modification gave us the most on target reads (50180 reads) for BRCA1 (198kb target) in both cell lines (Table 1). While the MCF 10A run did not give us target spanning long reads (Supplemental Fig. 1D), the SK-BR-3 ACME run gave us a $167 \mathrm{~kb}$ read (Fig. 2C).

The number of reads on target, irrespective of the nCATS modification used, were not sufficient to accurately call SVs in our region of interest. Based on these results, it seemed likely that a $200 \mathrm{~kb}$ target size may have been too large a region to enrich for using this method, as evidenced by the clear drops in reads around the middle of the target region.

Table 2. COSMIC database genes with known SVs in SK-BR-3

\begin{tabular}{|c|c|c|c|}
\hline Gene & Location & Gene size & Target size \\
\hline MYC & $8 q 24.21$ & 7517 & 12565 \\
\hline HOXA9 & $7 p 15.2$ & 12745 & 18506 \\
\hline FGFR4 & $5 q 35.2$ & 11270 & 19916 \\
\hline STK11 & $19 p 13.3$ & 22636 & 30286 \\
\hline CDKN2A & $9 p 21.3$ & 27291 & 30774 \\
\hline TERT & $5 p 15.33$ & 41880 & 44787 \\
\hline KRAS & $12 p 12.1$ & 46214 & 50955 \\
\hline BRCA2 & $13 q 13.1$ & 84192 & 91218 \\
\hline PAX7 & $1 p 36.13$ & 117860 & 120934 \\
\hline APC & $5 q 22.2$ & 138734 & 144265 \\
\hline \multicolumn{3}{|c|}{ Total target size } & $\mathbf{5 6 4 2 0 6}$ \\
\hline
\end{tabular}

Target size represents the shortest distance between crRNAs targeting the same gene
Cancer Gene Panel

To identify the largest optimal region size that could be entirely spanned with single contiguous reads, we designed a size titration experiment to target a panel of genes belonging to different size ranges (Table 2). These genes are present in the Catalogue Of Somatic Mutations In Cancer (COSMIC) database 21,22 and have known SVs in the HER-2-amplified breast cell line SK-BR-3 that were previously identified through whole genome long- and shortread sequencing $19,23,24$. Target sizes ranged from $10 \mathrm{~kb}$ to $150 \mathrm{~kb}$ with a total targeted region of $\sim 550 \mathrm{~kb}$ (Supplemental Table 1).

Given the improvement in the number of on-target bases and enrichment through pooling as well as background reduction, we further evaluated the performance of these modifications in targeting our cancer panel. Though iterative, Circulomics SRE has defined size cutoffs, making it a less amenable approach for background reduction when targets, such as our cancer panel, fall across different size groups. Since ACME relies on Cas9 bound nontarget DNA for effective background reduction, including more targets and therefore more crRNA
cos and better pulldown of background fragments. 


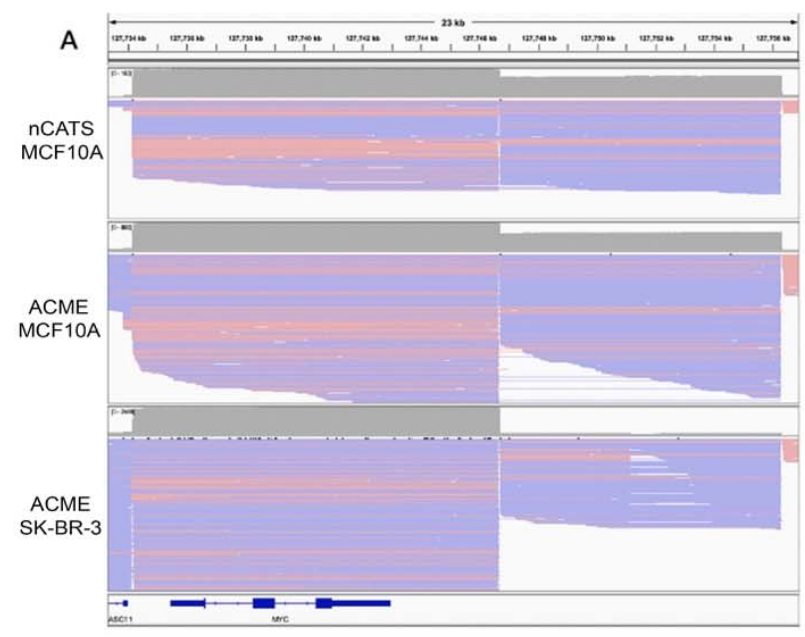

B
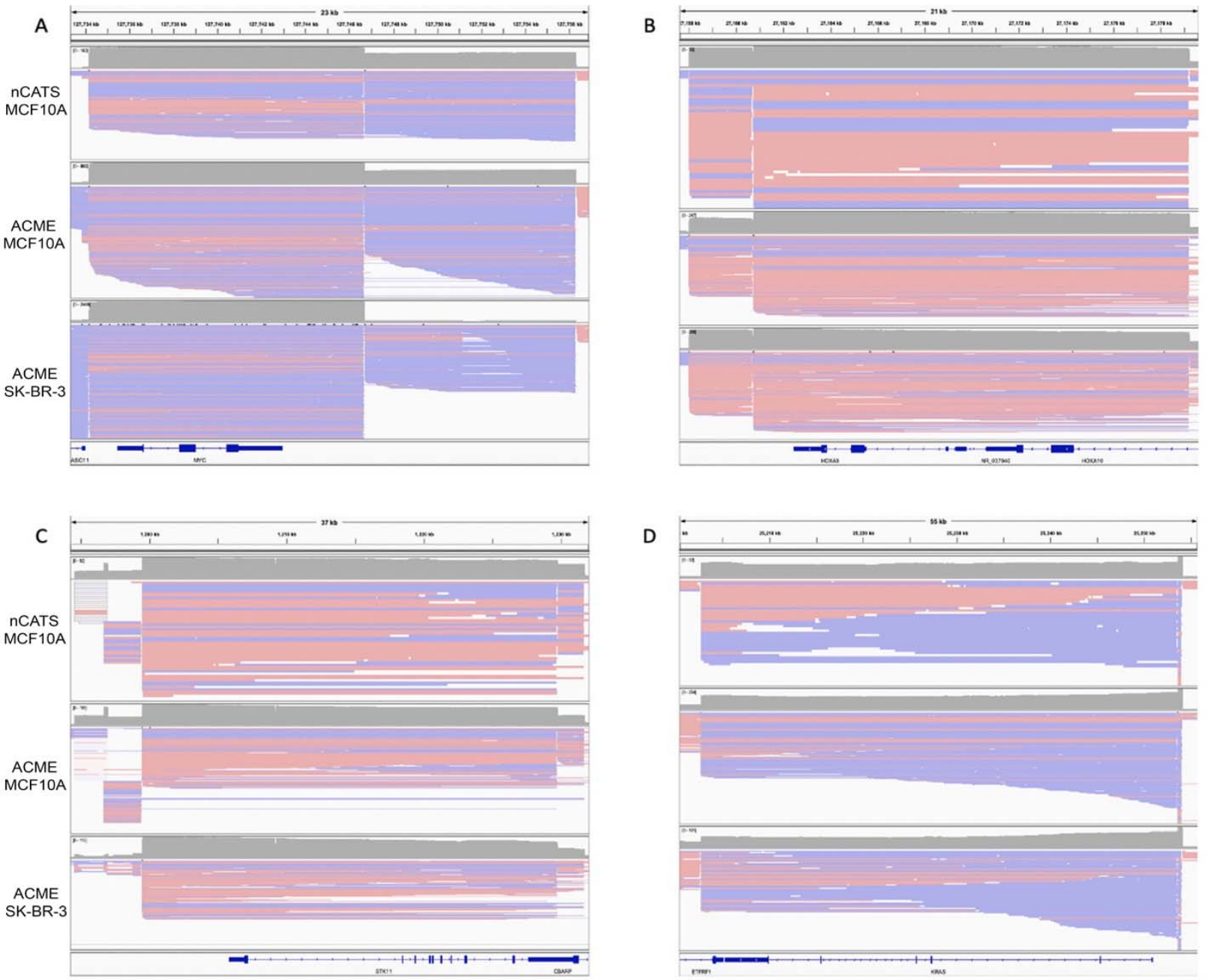

D
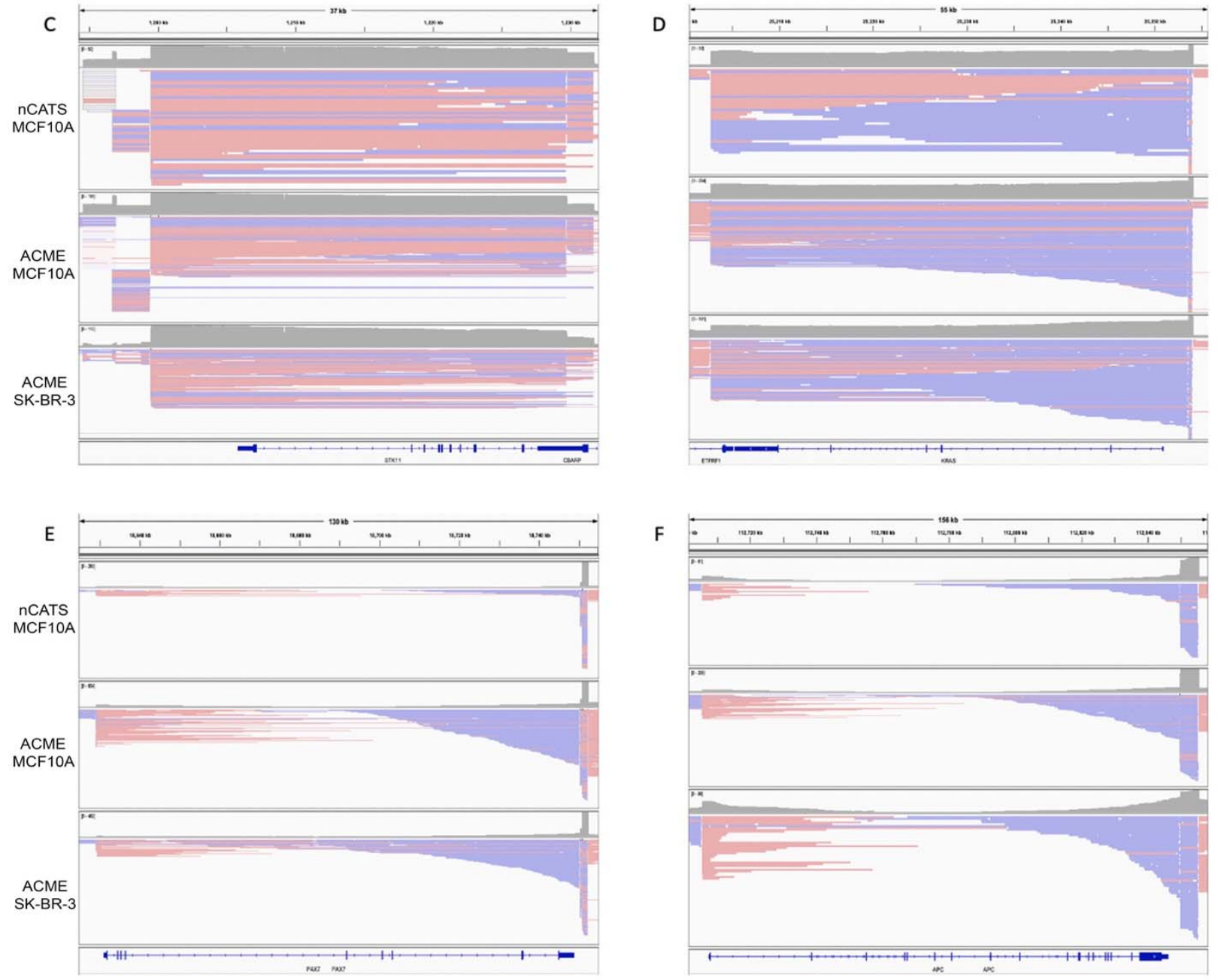

$\mathrm{F}$

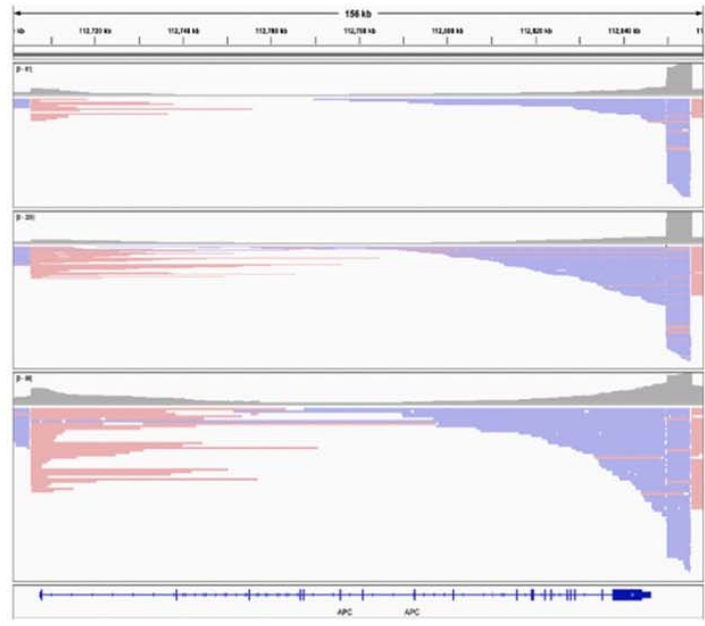

Fig. 3 IGV plots showing a representative gene from each size range across both cell lines MCF 10A and SK-BR-3. Plus strand reads are shown in pink and minus strand reads in blue. These genes were part of the 10 gene panel. For each image, the top panel shows reads that mapped to the gene in an MCF 10A library prepped without the ACME step (i.e., nCATS only); middle panel shows MCF 10A library prepped with the ACME step; bottom panel shows SK-BR-3 library prepped with ACME. Genes shown are A: MYC 10kb B: HOXA9 20kb C: STK11 30kb D: KRAS 50kb E: PAX7 120kb and F: APC 150 kb 
Table 3. Run summaries for BRCA2 from the different targeted cancer panel sequencing runs

\begin{tabular}{|c|c|c|c|c|}
\hline BRCA2 & $\begin{array}{c}\text { Single } \\
\text { MCF10A }\end{array}$ & $\begin{array}{c}\text { Pooled } \\
\text { MCF10A }\end{array}$ & $\begin{array}{c}\text { ACME (Pooled) } \\
\text { MCF10A }\end{array}$ & $\begin{array}{c}\text { ACME (Pooled) } \\
\text { SKBR3 }\end{array}$ \\
\hline On target bases & 326143 & 299552 & 5052621 & 3226173 \\
\hline On target reads & 9 & 12 & 135 & 115 \\
\hline Total bases & 190609890 & 276025707 & 2409003747 & 1894903771 \\
\hline Total reads & 10299 & 17281 & 111274 & 102727 \\
\hline Fold Enrichment & 101 & 41 & 86 & 62 \\
\hline Coverage & $4 x$ & $3 x$ & $55 x$ & $35 x$ \\
\hline
\end{tabular}

For modifications where more than one run was performed during the testing phase, average values are represented in the table.
As seen in Supplemental Table 2, all targets show a significant improvement in fold enrichment and coverage with the incorporation of ACME. Fig. 3 shows reads that mapped to some representative genes of the panel in both MCF $10 \mathrm{~A}$ and SK-BR-3 as viewed using IGV ${ }^{25}$. Smaller genes on the panel like MYC (12kb, Fig. 3A) and HOXA9 (18kb, Fig. 3B), and mid-sized genes like STK11 (30kb, Fig. 3C) had decent depth even without ACME, but their coverage almost doubles with the incorporation of ACME. Larger gene targets such as KRAS (50kb, Fig. 3D) and BRCA2 (90kb, Fig. 4), had poor coverage using nCATS. On using ACME, they not only have better coverage but also have significantly more target spanning reads that capture these genes in their entirety. Additionally, we see a drop in coverage around the middle of the target region for the two largest genes in the panel - PAX7 (120kb, Fig. 3E) and $A P C$ (145kb, Fig. 3F), indicating an upper limit of $100 \mathrm{~kb}$ for efficient targeting using ACME. Looking specifically at BRCA2 (Table 3), an important breast cancer gene, we see $60-90$-fold enrichment of this $91 \mathrm{~kb}$ target across both cell lines, giving us a coverage of $35-55 x$ of the gene.

One major advantage that ACME offers over other long-read targeting approaches is the ability to get multiple contiguous reads that span the entire length of the region of interest. For all genes on our panel that were $<=100 \mathrm{~kb}$ in size, we were able to obtain 3-7 times as many complete target spanning reads in comparison to the same regions captured using nCATS (Table 4). Even for larger targets, like BRCA2 and PAX7, we obtained 2-20 reads spanning the

Table 4. Number of reads spanning $X \%$ of gene for each targeted cancer panel gene across non-ACME and $A C M E$ pooled library sequencing runs

\begin{tabular}{|c|c|c|c|c|c|c|c|c|c|c|c|c|c|}
\hline & & & F10A & OACl & & & $\overline{\text { CF10A }}$ & $\overline{\mathrm{ACME}}$ & & & SKBR & ACME & \\
\hline Gene & Size & $100 \%$ & $80 \%$ & $60 \%$ & $40 \%$ & $100 \%$ & $80 \%$ & $60 \%$ & $40 \%$ & $100 \%$ & $80 \%$ & $60 \%$ & $40 \%$ \\
\hline MYC & 12565 & 129 & 140 & 148 & 153 & 993 & 1036 & 1055 & 1075 & 2057 & 2210 & 2300 & 2424 \\
\hline HOXA9 & 18506 & 61 & 63 & 68 & 76 & 425 & 444 & 471 & 489 & 47 & 106 & 118 & 133 \\
\hline FGFR4 & 19916 & 36 & 39 & 41 & 43 & 233 & 242 & 253 & 263 & 186 & 206 & 227 & 239 \\
\hline STK11 & 30286 & 29 & 30 & 35 & 40 & 152 & 161 & 168 & 183 & 64 & 79 & 89 & 102 \\
\hline CDKN2A & 30774 & NA & NA & NA & NA & NA & NA & $\mathrm{NA}$ & NA & 35 & 41 & 54 & 60 \\
\hline TERT & 44787 & 7 & 9 & 9 & 14 & 61 & 74 & 87 & 119 & 0 & 6 & 7 & 106 \\
\hline KRAS & 50955 & 9 & 13 & 19 & 26 & 111 & 123 & 142 & 168 & 27 & 37 & 43 & 60 \\
\hline BRCA2 & 91218 & 1 & 1 & 2 & 3 & 20 & 26 & 36 & 54 & 3 & 10 & 21 & 34 \\
\hline PAX7 & 120934 & 1 & 1 & 1 & 4 & 2 & 4 & 7 & 26 & 1 & 3 & 7 & 18 \\
\hline APC & 144265 & 0 & 0 & 0 & 3 & 0 & 1 & 2 & 16 & 0 & 1 & 1 & 7 \\
\hline
\end{tabular}

For some conditions, more than one run was performed during the testing phase. For these runs, average values are represented in the table. ${ }^{*}$ For one of the three MCF10A ACME runs, only 3 samples were pooled together instead of 4 . All other runs had 4 samples pooled together for each run. 
entire $90-120 \mathrm{~kb}$ targets, whereas these counts were between 0 to 2 reads for the $\sim 100 \mathrm{~kb}$ targets without ACME.

We also prepared

ACME enriched

single libraries

using just $5 \mu \mathrm{g}$

each of MCF $10 \mathrm{~A}$

and SK-BR-3 DNA.

On comparing

these results with a

single sample

nCATS run without

ACME

(Supplemental

Table 3), we see a significant increase

in coverage using ACME, especially for the larger genes on the panel. As seen with the pooled runs, ACME also helps increase the number of single contiguous reads spanning the entire target (Supplemental Table 4). This is evident from Supplemental
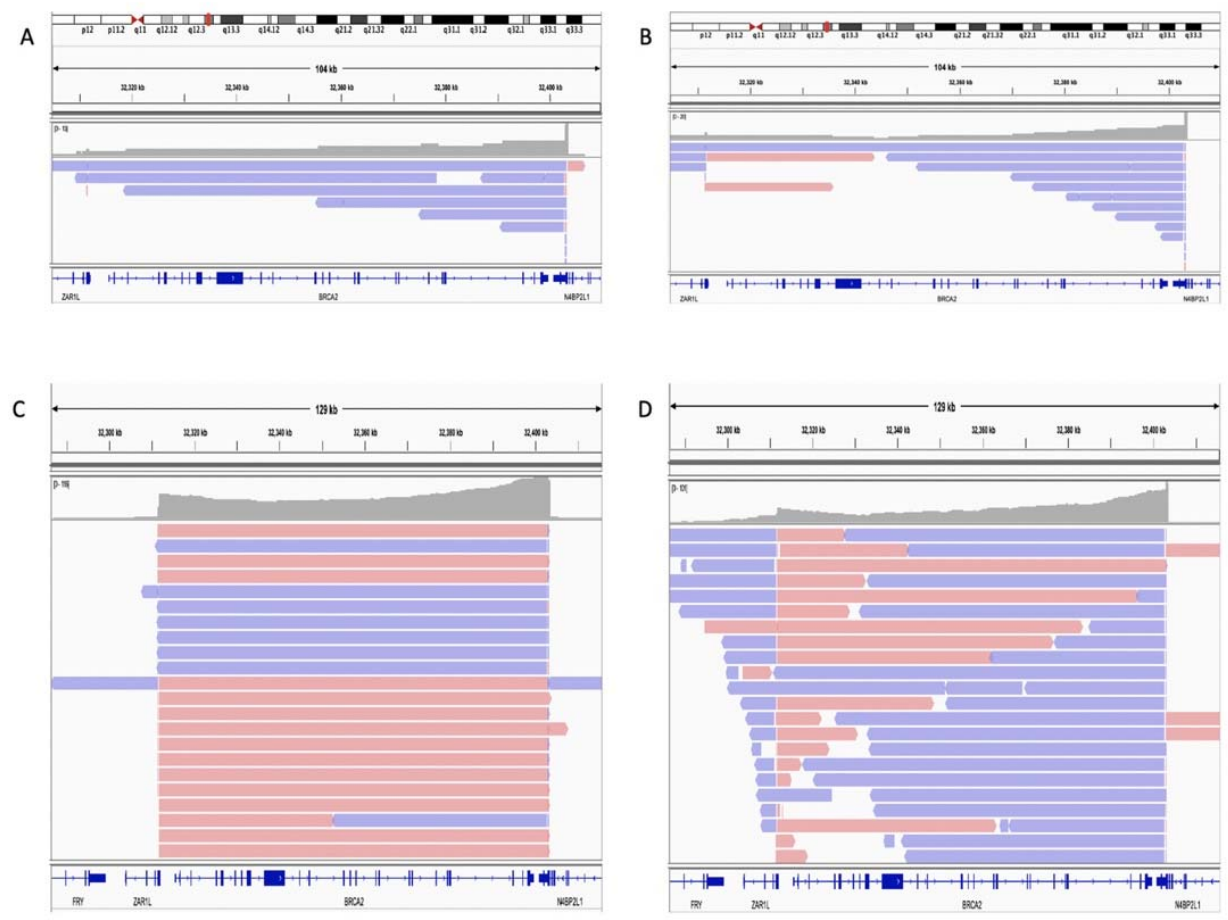

Fig 4. IGV plots showing reads that mapped to the $B R C A 2$ gene in different Cas9-mediated targeting libraries. Plus strand reads are shown in pink and minus strand reads in blue. A: Single sample library prep using MCF 10A DNA, without the ACME step (nCATS only) B: Library prepared by pooling together 3 identical library preps of MCF 10A DNA without the ACME step (nCATS only). C-D: Pooled library prepared from 4 identical preps of MCF $10 A$ and SK-BR-3 DNA resp. with the incorporation of the ACME step to remove non-target DNA.

Fig. 2, which

shows reads that mapped to a few representative genes from each size range on the panel. We clearly see that without ACME we lose the ability to capture reads that span the full length of the target in genes larger than $30 \mathrm{~kb}$, thereby limiting our scope of targeting these genes in rare samples.

\section{Structural variants}

Having identified ACME as an effective targeted long-read method, we evaluated its performance in detecting SVs in SK-BR-3. While MCF $10 A$ is a normal breast cell line ${ }^{18}$, SKBR-3 is a widely studied HER2+ breast cancer cell line ${ }^{19,24}$. Furthermore, we have previously carried out whole genome sequencing of this cell line using short-read paired-end Illumina, longread PacBio sequencing, 10X Genomics Linked Reads, and Oxford Nanopore sequencing ${ }^{23,26}$ allowing us to compare ACME's ability to detect variants with that of these widely used whole genome platforms. For ACME, SVs were called using Sniffles ${ }^{7}$ and merged both manually and by using SURVIVOR ${ }^{27}$, with the maximum distance between SVs set to 1000 bp and a minimum SV size of $20 \mathrm{bp}$, leaving us with 17 ACME SV calls. For the whole genome platforms, Sniffles and PBSV (https://github.com/PacificBiosciences/pbsv) were used to call SVs from ONT and PacBio datasets, while SVaBA ${ }^{28}$, Lumpy ${ }^{29}$, Manta ${ }^{30}$, GROC-SVs ${ }^{31}$, NAIBR ${ }^{32}$, and LongRanger ${ }^{33}$ were used on the Illumina/10X data to call SVs ${ }^{23,26}$. We subsetted SVs inferred 
from whole genome data across each sequencing platform to include only those that appeared within our target coordinates for each of the ten genes on our panel, giving us 11 ONT SVs and 15 PacBio SVs. For Illumina, SVs supported by at least 2 of 6 callers used by Aganezov et al. were included, accounting for Illumina's lower accuracy in SV detection and to reduce the false positives that are known to arise on inferring SVs from short-read data.

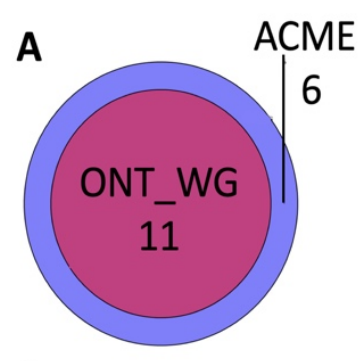

C

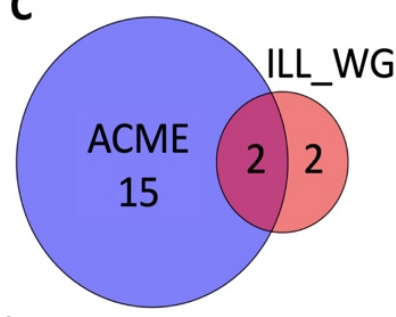

Fig. 5. Comparison of pooled ACME with whole genome platforms for SV detection in SK-BR-3. AC: SVs detected by ACME that overlap with ONT, Pacific Biosciences (PB), and Illumina (ILL) whole genome sequencing (WG) resp. D: High concordance observed between PB and ONT WG for SV detection in SK-BR-3. Note: Only SVs appearing within our target coordinates for each of the ten genes on our panel were included in these comparisons. SVs were detected from pooled sample prep ACME run.

Overlaps between ACME and each whole genome SV callset were inferred based on their positions, such that an SV from the ACME callset that occurred within $1000 \mathrm{bp}$ of an SV from a whole genome callset overlapped and were considered the same event. The same approach was used to determine overlaps between the different whole genome SV callsets. We observed very strong concordance between ACME SVs with those from whole genome ONT and PacBio (Fig. 5). ACME successfully identified all SVs from the PacBio and ONT datasets that occurred within our target regions (Supplemental Table 5). Additionally, ACME inferred 2 SVs that were not called by PacBio, and 6 SVs that were not called by ONT, which could be attributed to the higher on target coverage achieved by ACME. Of the 4 Illumina SVs, only 2 events overlapped with each ACME, PacBio, and ONT SV callsets, and were common between all platforms. Our results highlight that ACME performs similar to ONT and PacBio whole genome sequencing for comprehensive SV inference thus establishing it as a viable and effective targeted long-read approach. We also looked at ACME's ability to detect SVs from a single sample prep with initial DNA input of $5 \mu \mathrm{g}$. ACME was able to identify 12 SVs from the single library data compared to the 17 SV calls from the pooled library, with 9 overlaps with ONT SV calls and 12 overlaps with PB SV calls. (Supplemental Fig. 3)

\section{Single Nucleotide Polymorphisms}

Nanopore sequencing tends to have low sensitivity in SNP detection due to its difficulty in distinguishing signal events in repetitive regions like homopolymers. We explored how the increased coverage using ACME would improve the ability to call SNPs from the SK-BR-3 dataset. We used SNP calls from the short-read paired-end Illumina dataset, and also re-ran SNP callers on our previously sequenced long-read PacBio and Oxford Nanopore datasets ${ }^{23,26}$ to look for variants common between the platforms.

All SNP callsets were filtered to include only those variants that fell within our target regions. xAtlas ${ }^{34}$ was used to call SNPs from the lllumina data giving us 500 in-target SNPs. Clair ${ }^{35}$ was 
used to call SNPs from the whole genome ONT (1234 SNPs) and PacBio (601 SNPs) datasets and the targeted ACME dataset (592 SNPs).

For the first round of comparisons between platforms, we looked at all SNPs that met a minimum depth of $>=10$ for each platform. Assuming Illumina as the gold standard for SNP detection, we looked for SNPs from each long-read platform that overlapped with SNPs called from the Illumina dataset. As seen in Fig. 6 ACME performed on par with the whole genome long-read platforms when compared to Illumina for SNP detection. We also noted a high number of ONT whole genome SNPs that did not overlap with Illumina (Fig. 6A). This could be explained by the overall lower depth of the ONT WG data and the older basecaller used on this dataset, which produced lower single read accuracy and could therefore generate more false positives due to basecall errors. In contrast, we observed that the higher depth from
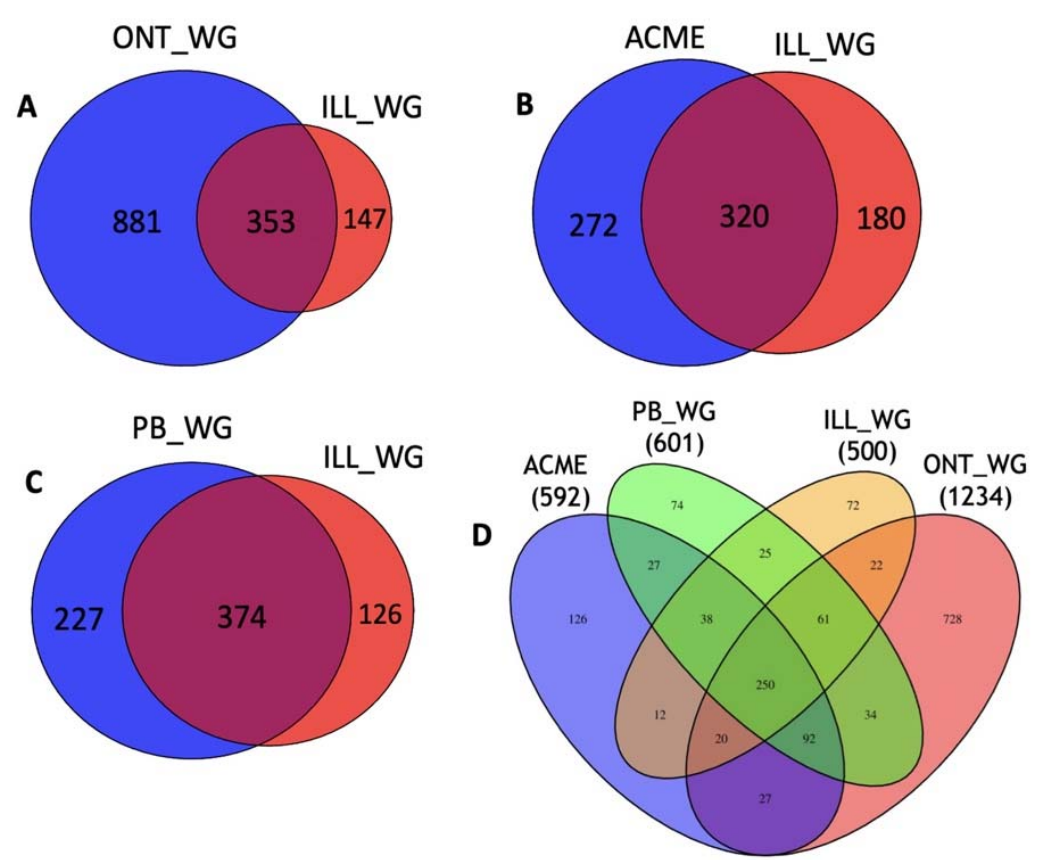

Fig. 6. Comparison of long-read platforms with Illumina whole-genome sequencing for SNP detection in SK-BR-3. A-C: SNPs detected by Illumina (ILL) that overlap with ONT, ACME, and Pacific Biosciences (PB) resp. D: SNPs detected by and that overlap between each platform. Note: Only SNPs with minimum depth of $10 x$ appearing within our target coordinates for each of the ten genes on our panel were included in these comparisons. SNPs were detected from pooled sample prep ACME run. targeted ACME (Fig. 6B), called with the latest basecaller, helped bring down the number of false positive SNPs called by the nanopore platform. On performing the same set of comparisons using data generated from a single library prep of ACME ( $5 \mu \mathrm{g}$ initial DNA input), we observed that of the 337 SNPs inferred by ACME, 151 overlap with Illumina. (Supplemental Fig. 4).

Given the lack of coverage across the full length of targets $>100 \mathrm{~kb}$ in the ACME dataset, we reran the above comparisons between platforms (minimum depth $>=10$ ) after filtering out SNPs present in the PAX7 ( 120kb) and APC ( 150kb) genes across all platforms. We observed an increase in the percentage of ACME-ILL overlapping SNPs from $64 \%$ to $76 \%$. The overlaps between ONT-ILL and PB-ILL however remained consistent (Supplemental Fig. 5). This indicates that ACME is as capable as, if not better than, the whole genome long-read platforms for SNP detection in regions with complete coverage of target without any dropouts in the middle.

Lastly, in addition to calling SNPs de novo as described thus far, we also looked at forced genotype calls using the Illumina SNP set as the query. A forced genotype approach is particularly useful for detecting specific SNPs of interest in long-read datasets. Through the 
forced genotype approach, we specifically looked for 618 SNPs called from the Illumina dataset (minimum depth $>=6$ ) in our long-read datasets to see if these platforms detected the query SNPs irrespective of their depth. We found that ACME detected $88 \%$ of SNPs in the query set, again performing on par with PacBio whole genome (90\% Illumina SNPs detected) and ONT whole genome (87\% Illumina SNPs detected). Additionally, we observed that forced genotype calls generated from the single library ACME run were also largely comparable, with $82 \%$ of Illumina SNPs detected (Supplemental Fig. 6).

\section{Discussion:}

Whole genome, long-read sequencing is currently expensive and too low throughput for largescale variant studies. Targeted DNA sequencing is a cost-effective approach that facilitates higher sample throughput and improves accuracy, giving depths necessary to detect rare alleles in a heterogenous population of cells. In this study, we developed an Affinity-based $\underline{\text { Cas9- }}$ Mediated Enrichment (ACME) approach, that builds upon the existing nCATS method by adding a simple background reduction step, physically increasing the on-target to off-target ratio in the sample prior to sequencing. Unlike exome capture, ACME helps capture entire gene regions, including introns, as single sequencing reads up to $100 \mathrm{~kb}$ in size. ACME leverages the presence of a 6-histidine tag at the C-terminal domain of the Cas9 nuclease that is used for cleaving and releasing the targets from the dephosphorylated DNA. Since Cas9 remains preferentially bound to the non-target side of the cleavage site, using HisTag isolation and pulldown we were able to physically remove Cas9-bound non-targets from the sequencing pool prior to adapter ligation. Using ACME, we captured and sequenced fragments as large as $100 \mathrm{~kb}$ with 35-55x coverage, doubling the existing single read sizes capturable. By eliminating smaller competing non-targets from the sequencing library, we saw a 3- to 7-fold increase the number of whole gene spanning reads per target, thereby aiding in SV discovery by reducing potential mapping errors.

Compared to traditional nCATS, we find that ACME not only helps in increasing target coverage but also allows for the complete spanning of genes by generating reads as long as $100 \mathrm{~kb}$ - up to 3 times the targetable size and read lengths achieved using nCATS. We have shown that even for $90-120 \mathrm{~kb}$ sized targets (BRCA2 and PAX7), ACME helped capture 2-20 reads spanning the entire targets, whereas without ACME these counts were between 0 to 2 reads. We ran the nCATS and ACME experiments parallelly using the same HMW DNA, since DNA quality is important for optimal targeting and generating long reads. Our results therefore specifically highlight the advantage offered by ACME's ability to reduce competing background reads. Two other Cas9-based targeting approaches that involve background reduction - CaBagE ${ }^{13}$ and Negative Enrichment ${ }^{14}$ - use exonuclease digestion to eliminate non-target DNA. Both studies have only successfully achieved target read lengths of $<35 \mathrm{~kb}$ in size and pose the additional risk of digesting nicked target fragments that are not protected by Cas 9 binding. Furthermore, the exonuclease step adds $2-4$ hours to the library prep, while the ACME step adds $<45$ mins, making it a quick and efficient targeted long-read approach.

We demonstrated ACME's ability to detect variants by comparing it with PacBio, ONT, and Illumina whole-genome sequencing of SK-BR-3. For SVs, we showed that ACME detected all SVs within our targets that were previously inferred by PacBio and ONT whole-genome sequencing ${ }^{23,26}$. ACME's higher coverage also helped identify a few novel SVs previously undetected by whole genome PacBio and ONT, which we are currently validating. Since the limitations for SV detection with long-read technologies are largely cost driven, we show that ACME performs on par with not only the more expensive PacBio platform, but also surpasses depths achievable by ONT whole-genome sequencing. For SNPs, we found that ACME performs similar to whole genome PacBio and ONT when we compared the long-read platforms 
to Illumina, which is considered the gold standard. To perform a fairer comparison between platforms, we excluded SNPs from $P A X 7$ and $A P C$ across all platforms on finding coverage dropouts in targets $>100 \mathrm{~kb}$ in the ACME callsets. We then found that ACME not only matches but surpasses the long-read platforms in their ability to detect SNPs. ACME detected more SNPs called by Illumina than the whole-genome PB and ONT, likely due to increased target coverage. We also found that ACME performs on par with these platforms when we adopted the forced genotype approach. These results are promising as they show that ACME can effectively replace the whole genome long-read platforms for SNP detection by offering better depth and increased sensitivity at a fraction of the cost.

While pooled ACME runs start with $20 \mu \mathrm{g}$ of initial DNA input, we also evaluated the performance of single library ACME preps starting with $5 \mu \mathrm{g}$ to account for the realistic possibility of having limited sample DNA available. For SV detection, we observed that ACME still performs better than ONT whole-genome sequencing, while performing on par with PacBio by failing to detect only $3 \mathrm{SVs}$ found by PacBio. For SNPs, the number of ACME-Illumina overlaps fell drastically on switching from pooled to single ACME preps. This drop in variant calls, especially SNPs, may be attributed to the lower coverage observed in the single library data. While all experiments described in this study were run using the early access developer protocol (ONT protocol versions ENR_9084_v109_revB_04Dec2018 and ENR_9084_v109_revH_04Dec2018) that required sourcing individual reagents separately, with the release of the standalone ONT Cas9 kit (SQK-CS9109), coverage from single library preps is no longer a concern. We tested the CS9109 kit performance with and without ACME across different preps and concluded that this newer kit offers improved yield and a much higher coverage for the single as well as pooled library preps than previously observed. Our efforts to run ACME on the low pore Flongle device have been unsuccessful and ONT has withdrawn support for nCATS on the Flongle.

Adaptive sequencing approaches like ReadFish ${ }^{15}$ and UNCALLED ${ }^{16}$ can target 100 s of regions per run, however increased rejection of reads affects sequencing yield, leads to quicker pore burnouts, and lower target coverage $(<60 x$, usually close to $20-30 x)$ than enzymatic methods can achieve. Since sheared DNA (8-15kb) is required for these approaches, it is better suited to detect single-nucleotide variants (SNVs), copy number variants (CNVs), and repeat expansions than detecting larger rare variants ${ }^{36}$. On evaluating if ACME could be combined with adaptive sequencing to play to both their strengths, we found that adaptive sequencing offers no enrichment, and in fact negatively impacts enzymatic enrichment, for panels with few targets $(<0.1-1 \%$ of the genome). Therefore, while adaptive sequencing may not be the best tool for discovery of rare or large variants, it is great for clinical settings ${ }^{36}$ to survey 100 s of targets for known variants, which can then be confirmed with higher depth enzymatic approaches such as nCATS or ACME.

A current limitation of ACME is the inability to sequence deeply targets $>100 \mathrm{~kb}$ in size without coverage dropouts in the center. However, this could be a function of DNA size more than the targeting approach itself and may be solved by switching to the recently released Ultra HMW (UHMW) DNA Extraction protocol from Circulomics (Pacific Biosciences). Moreover, even with the $100 \mathrm{~kb}$ size limit ACME offers distinct advantages over the other Cas9 based targeting approaches. Targets $>100 \mathrm{~kb}$ can be captured using ACME with the designing of tiled guides, with tiles spanning $50-80 \mathrm{~kb}$ regions as opposed to $20-30 \mathrm{~kb}$ with the other approaches. This will not only reduce the number of tiles per target, but also the crRNA guides per reaction, allowing for more targets to be captured per reaction. The main advantage ACME offers over other amplification free long-read targeting approaches is the ability to capture several contiguous 
reads, up to $100 \mathrm{~kb}$ in size, that span the whole target from start to end. This helps in reducing mapping errors and aid in SV detection even with lower target coverage for large gene targets.

\section{Methods:}

\section{Cell culture and DNA extraction}

MCF 10A and SK-BR-3 cell lines were obtained from ATCC and cultured as per standard guidelines. High Molecular Weight (HMW) DNA was extracted using an adaptation of a modified Sambrook and Russell ${ }^{37}$ DNA extraction described by Jain et al. ${ }^{38}$ Approximately $3 \times 10^{7}$ cells were spun at $150 \mathrm{rpm}$ for $10 \mathrm{~min}$ to pellet. The cells were resuspended in $200 \mu \mathrm{l}$ PBS. $10 \mathrm{ml}$ TLB was added (100 mM NaCl, $10 \mathrm{mM}$ Tris-Cl pH 8.0, $25 \mathrm{mM}$ EDTA pH 8.0, 0.5\% (w/v) SDS, $20 \mu \mathrm{g} / \mathrm{ml}$ Qiagen RNase A), vortexed at full speed for $5 \mathrm{~s}$ and incubated at $37^{\circ} \mathrm{C}$ for $1 \mathrm{hr} .100 \mu \mathrm{l}$ Proteinase K (Qiagen, final conc. $200 \mu \mathrm{g} / \mathrm{ml}$ ) was added and mixed by slowly rotating end over end three times followed by $2 \mathrm{hr}$. incubation at $50^{\circ} \mathrm{C}$ with gentle mixing every $30 \mathrm{mins}$. The lysate was split into two, phenol-purified using $5 \mathrm{ml}$ phenol-chloroform-isoamyl alcohol (25:24:1), mixed end over end for $10 \mathrm{~min}$, and spun at $4000 \mathrm{rpm}$ for 10 mins. Supernatant was transferred to fresh tubes using wide bore tips, $5 \mathrm{ml}$ chloroform-isoamyl alcohol (24:1) was added, mixed end over end for $10 \mathrm{~min}$, and spun at $4000 \mathrm{rpm}$ for $10 \mathrm{mins}$. Using wide bore tips, supernatants were combined and DNA was precipitated by the addition of $4 \mathrm{ml} 5 \mathrm{M}$ ammonium acetate and $30 \mathrm{ml}$ ice-cold $100 \%$ ethanol followed by gentle rocking for $15 \mathrm{~min}$. DNA was recovered with a glass hook followed by washing twice in $70 \%$ ethanol. After spinning down at $10,000 \mathrm{~g}$, ethanol was removed followed by air drying for $15 \mathrm{~min}$. $300 \mu \mathrm{l}$ of nuclease free water was added to the DNA and left at $4^{\circ} \mathrm{C}$ overnight to resuspend. DNA was quantified using the Qubit fluorometer (ThermoFisher Scientific) and Femto Pulse System (Agilent) before use.

\section{Guide RNA design}

crRNA probes were designed to target and cut on either side of the region of interest (ROI) to excise that region. Four to six crRNA guides were designed per target for redundancy - 2-3 upstream of the ROI targeting the (+) strand and 2-3 downstream that targeted the (-) strand. Guides were designed using CHOPCHOP (http://chopchop.cbu.uib.no/) ${ }^{39,40}$ and chosen based on efficiency $(>0.3)$, GC content $(40-80 \%)$, self-complementarity (score $=0$ ), and predicted mismatches $(<5)$, with at least $1 \mathrm{~kb}$ of flanking sequence either side of the ROI. Duplex format of guide RNAs were ordered, comprising of synthetic crRNAs (IDT Cas9 Alt-R ${ }^{\mathrm{TM}}$ crRNAs, custom designed) and tracrRNAs (IDT Alt-R ${ }^{\mathrm{TM}}$, Cat \#1072532). Guide sequences for all genes in the panel are provided in Supplementary Table 1.

\section{RNP assembly and Cas9 cleavage}

All crRNAs for all genes in the panel were resuspended at $100 \mu \mathrm{M}$ in TE (pH 7.5) and pooled into an equimolar mix prior to guide RNA assembly. As described in Gilpatrick et al. ${ }^{41}$ and using ONT protocol versions ENR_9084_v109_revB_04Dec2018 and ENR_9084_v109_revH_04Dec2018 for reference, crRNA ${ }^{-}$mix and tracrRNA were combined such that the tracrRNA and total crRNA concentration were both $10 \mu \mathrm{M}$. gRNA duplexes were formed by denaturation at $95^{\circ} \mathrm{C}$ for $5 \mathrm{~min}$ and then cooling to room temp for $5 \mathrm{~min}$. Ribonucleoprotein complexes (RNPs) were constructed by combining $10 \mu \mathrm{l}$ of annealed crRNA•tracrRNA pool $(10 \mu \mathrm{M})$ with $0.8 \mu \mathrm{l}$ of HiFi Cas9 Nuclease V3 (62 $\mu \mathrm{M}$, IDT Cat \#1081060) in $10 \mu \mathrm{l}$ of $10 \mathrm{X}$ CutSmart Buffer (NEB, Cat \#B7204) at a final volume of $100 \mu \mathrm{L}$, incubated for 30 min at room temperature, then stored on ice until use. This mix is good for 10 reactions and assembled RNPs can be stored at $4^{\circ} \mathrm{C}$ for up to a week. Genomic DNA was dephosphorylated by adding $3 \mu \mathrm{l}$ of Quick CIP enzyme (NEB, Cat \#M0508) to $5 \mu \mathrm{g}$ of input DNA (in total volume of $24 \mu \mathrm{l}$ at $>210 \mathrm{ng} / \mu \mathrm{l}$ ) with $3 \mu \mathrm{l}$ 10X CutSmart Buffer (NEB, Cat \#B7204), and incubating for 10 min at $37^{\circ} \mathrm{C}$, followed by heating for $2 \mathrm{~min}$ at $80^{\circ} \mathrm{C}$. After allowing the sample to return to room 
temp, $10 \mu \mathrm{L}$ of the pre-assembled Cas9 RNP was added to the sample. In the same tube, $1 \mu \mathrm{L}$ of $10 \mathrm{mM}$ dATP (NEB, Cat \# N0440S) and 1uL of Taq DNA polymerase (NEB, Cat \#M0273) were added for A-tailing of DNA ends. The sample was then incubated at $37^{\circ} \mathrm{C}$ for 15 min for Cas 9 cleavage followed by $5 \mathrm{~min}$ at $72^{\circ} \mathrm{C}$ for A-tailing. For pooled libraries, four identical preps of $5 \mu \mathrm{g}$ each were prepared parallelly and taken to the next step. For single libraries, only 1 prep of $5 \mu \mathrm{g}$ was carried forward to the next step.

\section{nCATS modifications for BRCA1 targeting \\ - Pooled Library Preparation:}

For each sequencing run, four reactions were set up starting with $5 \mu \mathrm{g}$ HMW DNA for each reaction, giving a total of $20 \mu \mathrm{g}$ starting material across the 4 reactions. Each reaction was taken though the steps on nCATS individually until the adapter ligation step, wherein each product was eluted to a lower volume and pooled together at the end before loading onto the flowcell. In order to avoid overloading the flowcell, only half the library was loaded initially with the other half loaded 24 hours into the sequencing run.

\section{- $\quad$ Circulomics Short Read Elimination (SRE):}

Since the pooled library prep generated more on target reads, we started with four identical preps each of MCF 10 A and SK-BR-3 DNA. For each prep, SRE was performed immediately after the Cas9 cleavage and dA-tailing step. The products of SRE were cleaned up using $1 \mathrm{X}$ Ampure XP beads, eluted in a smaller volume, and pooled together respectively for both MCF $10 \mathrm{~A}$ and SK-BR-3.

- Affinity-based Cas9-Mediated Enrichment (ACME):

For both cell lines, four identical sample preps of $5 \mu \mathrm{g}$ HMW DNA each, were run through our modified nCATs protocol described in detail below. Like the SRE modification, products of the Cas 9 cleavage and $\mathrm{dA}$-tailing step were immediately processed using ACME to remove Cas9 bound non-target DNA from the sample. ACME products were cleaned up using $1 \mathrm{X}$ Ampure XP beads, eluted in a smaller volume, and pooled together respectively for both MCF 10A and SKBR-3.

\section{Affinity-based Cas9-Mediated Enrichment (ACME)}

For pooled ACME preps, four identical preps of $5 \mu \mathrm{g}$ each were set up for both MCF $10 \mathrm{~A}$ and SK-BR-3. crRNA guides for all 10 genes were pooled together into an equimolar mix for the Cas9 cleavage step. ACME was performed on each sample separately as follows: The $42 \mu l$ cleaved sample from the Cas9 cleavage and dA-tailing step was resuspended in $700 \mu$ of freshly prepared $1 \mathrm{X}$ binding/wash buffer (2X Binding/Wash Buffer: $100 \mathrm{mM}$ Sodium Phosphate $\mathrm{pH}$ 8.0, 600 mM NaCl, 0.02\% Tween ${ }^{\mathrm{TM}}$-20). Invitrogen ${ }^{\mathrm{TM}}$ His-Tag Dynabeads ${ }^{\mathrm{TM}}$ (Cat \#10103D) were thoroughly resuspended in the vial (vortexed $>30 \mathrm{sec}$ ), $50 \mu \mathrm{L}(2 \mathrm{mg})$ of which was transferred to a fresh $1.5 \mu \mathrm{l}$ Eppendorf DNA LoBind tube. Tube was placed on a magnet for 2 min, supernatant was discarded, and the sample (prepared in $1 \mathrm{X}$ binding/wash buffer) was added to the beads and mixed well. After incubating on a roller for 5-10 min at room temperature, the tube was placed on a magnetic rack for $2 \mathrm{~min}$, and the supernatant was carefully transferred to a fresh tube using wide bore tips. Note: The targets/regions of interest are suspended in the supernatant while the Cas9-bound non-targets are bound to the His-Tag beads. DO NOT discard the supernatant at this step. The sample was cleaned up using $1 \mathrm{X}$ Ampure XP beads (Beckman Coulter, Cat \#A63881) and eluted in nuclease-free water. Based on the number of initial parallel preps, the elution volume was adjusted to give a final volume of $44 \mu \mathrm{l}$ at the end of ACME i.e., eluted in $11 \mu \mathrm{l}$ each (for the pooled library) or $44 \mu \mathrm{l}$ (for single library). ACME products were quantified using the Qubit fluorometer (ThermoFisher Scientific). $42 \mu \mathrm{l}$ of the ACME enriched sample was taken forward to the adapter ligation step. 


\section{Library prep and sequencing}

Sequencing adaptors and ligation buffer from the Oxford Nanopore Ligation Sequencing Kit (ONT, LSK109) were ligated to cut DNA ends using T4 DNA ligase (NEBNext Quick Ligation Module E6056) for $10 \mathrm{~min}$ at room temperature. The sample was cleaned up using $0.3 \mathrm{X}$ Ampure XP beads (Beckman Coulter, Cat \#A63881) and washed twice with the long-fragment buffer (LFB; ONT, LSK109), resuspending the beads in the buffer each time. Sample was eluted in $15 \mu \mathrm{l}$ of elution buffer (EB; ONT, LSK109) for $30 \mathrm{~min}$ at room temperature. Note: Elution time of $30 \mathrm{~min}$ is recommended for targets $>30 \mathrm{~kb}$. Sequencing libraries were prepared by adding 25 $\mu \mathrm{L}$ sequencing buffer (SQB; ONT, LSK109) and $13 \mu$ loading beads (LB; ONT, LSK109) to 12 $\mu \mathrm{l}$ of the eluate. Each sample was run on a FLO-MIN106 R9.4.1 flow cell with $>1200$ active pores following Platform QC on the GridION sequencer. $30 \mu \mathrm{l}$ of flush tether (FLT; ONT, LSK109) was added to 1 tube of flush buffer (FB; ONT, LSK109). Initial flow cell priming was performed with $800 \mu \mathrm{L}$ of this mix and allowed 5 min to equilibrate. The flow cells were then primed with $200 \mu \mathrm{L}$ of the priming mix prior to loading the sequencing libraries.

\section{Analysis}

Nanopore reads were base called using Guppy v3.2. Reads were transferred to an isilon NL400 storage server and mapped to the hg38 human reference genome (UCSC Genome Browser) using either MiniMap2 ${ }^{42}$ or NGMLR ${ }^{7}$. The BAM files were sorted and indexed with Samtools ${ }^{43}$ and on-target reads were visualized using IGV ${ }^{25}$. Capture metrics including depth and breadth of target coverage and target enrichment were generated with Picard HSmetrics (https://broadinstitute.github.io/picard/index.html) and GATK DepthofCoverage ${ }^{44}$. Structural variants were called with Sniffles ${ }^{7}$. VCF files were sorted with VCFtools ${ }^{45}$. SNPs were called using Clair ${ }^{35}$. Variant comparisons between datasets and target-spanning read counts were done with BEDtools ${ }^{46}$.

- SV detection

Sniffles uses strand information to help confirm the event type as such: Strand orientation of the adjacency (DEL/INS:+-, DUP:-+, INV:++/--). During merging using SURVIVOR, SV types were not required to be the same, but strand/orientation was required to match. For ACME, ONT and PacBio, we did iterative filtering based on read support, which was required to be $>=4$ reads supporting the SV, and variant read counts, which was required to be $>=5 \%$ of total read counts.

\section{- SNP detection \\ SNP calling with $x$ Atlas on Illumina data:}

The "PASS" filters were engaged during SNP calling, initially with a minimum depth $>=6$, variant read depth $>2$, and a strand bias filter, giving us a total of 618 SNPs within our targets. Depth was then raised to $>=10$, giving us 500 SNPs that were used for the comparisons. The following additional filters were used for SNP calling with XAtlas on the lllumina data:

"low_snpqual", Description= "SNP logistic regression P-value is $>0.5$ "

"low_VariantReads", Description= "Variant read depth is $>2$ "

"low_VariantRatio", Description= "Variant read ratio is not less than Phred-scaled Genotype Likelihood scores cutoff"

"low_coverage", Description = "Total coverage is $>=6 "$

"high_coverage", Description= "Total coverage is $<=8000$ "

"single_strand", Description= "All variant reads are NOT in a single strand direction"

"No_data", Description= "At least 1 valid read on this site"

"No_var", Description= "At least 1 valid variant read on this site"

SNP calling with Clair on the long-read data: 
Minimum depth was set to $>=10$ with $10 \%$ alternate allele frequency and a filtration score of 400. Variants are divided into 10 categories: 1) a homozygous reference allele; 2) a homozygous 1 SNP allele; 3 ) a heterozygous 1 SNP allele, or heterozygous 2 SNP alleles; 4) a homozygous 1 insertion allele; 5) a heterozygous 1 insertion allele, or heterozygous 1 SNP and 1 insertion alleles; 6) heterozygous 2 insertion alleles; 7) a homozygous 1 deletion allele; 8) a heterozygous 1 deletion allele, or heterozygous 1 SNP and 1 deletion alleles; 9) heterozygous 2 deletion alleles; and 10) a heterozygous 1 insertion and 1 deletion alleles. The likelihood value of the 10 categories is calculated for each candidate variant, and the category with the largest likelihood value is chosen. The variant quality is calculated as the square of the Phred score of the distance between the largest and the second-largest likelihood values.

\section{References:}

1. Sudmant, P. H. et al. An integrated map of structural variation in 2,504 human genomes. Nature 526, 75-81 (2015).

2. Mills, R. E. et al. Mapping copy number variation by population-scale genome sequencing. Nature 470, 59-65 (2011).

3. Teo, S. M., Pawitan, Y., Ku, C. S., Chia, K. S. \& Salim, A. Statistical challenges associated with detecting copy number variations with next-generation sequencing. Bioinformatics 28, 2711-2718 (2012).

4. Lucas Lledó, J. I. \& Cáceres, M. On the Power and the Systematic Biases of the Detection of Chromosomal Inversions by Paired-End Genome Sequencing. PLoS One 8, e61292 (2013).

5. Eid, J. et al. Real-Time DNA Sequencing from Single Polymerase Molecules. Science (80-. ). 323, 133-138 (2009).

6. Clarke, J. et al. Continuous base identification for single-molecule nanopore DNA sequencing. Nat. Nanotechnol. 4, 265-270 (2009).

7. Sedlazeck, F. J. et al. Accurate detection of complex structural variations using singlemolecule sequencing. Nat. Methods 15, 461-468 (2018).

8. Audano, P. A. et al. Characterizing the Major Structural Variant Alleles of the Human Genome. Cell 176, 663-675.e19 (2019).

9. De Coster, W. et al. Structural variants identified by Oxford Nanopore PromethION sequencing of the human genome. Genome Res. 29, 1178-1187 (2019).

10. Dapprich, J. et al. The next generation of target capture technologies - large DNA fragment enrichment and sequencing determines regional genomic variation of high complexity. BMC Genomics 17, 486 (2016).

11. Stangl, C. et al. Partner independent fusion gene detection by multiplexed CRISPR-Cas9 enrichment and long read nanopore sequencing. Nat. Commun. 11, (2020).

12. Watson, C. M. et al. Cas9-based enrichment and single-molecule sequencing for precise characterization of genomic duplications. Lab. Investig. 100, 135-146 (2020).

13. Wallace, A. D. et al. CaBagE: A Cas9-based Background Elimination strategy for targeted, long-read DNA sequencing. PLoS One 16, (2021).

14. Stevens, R. C. et al. A novel CRISPR/Cas9 associated technology for sequence-specific nucleic acid enrichment. PLoS One 14, e0215441 (2019).

15. Payne, A. et al. Readfish enables targeted nanopore sequencing of gigabase-sized genomes. Nat. Biotechnol. 1-9 (2020). doi:10.1038/s41587-020-00746-X

16. Kovaka, S., Fan, Y., Ni, B., Timp, W. \& Schatz, M. C. Targeted nanopore sequencing by real-time mapping of raw electrical signal with UNCALLED. Nat. Biotechnol. 1-11 (2020). doi:10.1038/s41587-020-0731-9

17. Richardson, C. D., Ray, G. J., DeWitt, M. A., Curie, G. L. \& Corn, J. E. Enhancing homology-directed genome editing by catalytically active and inactive CRISPR-Cas9 using asymmetric donor DNA. Nat. Biotechnol. 34, 339-344 (2016). 
18. Soule, H. D. et al. Isolation and Characterization of a Spontaneously Immortalized Human Breast Epithelial Cell Line, MCF-10. Cancer Res. 50, (1990).

19. Fogh, J., Fogh, J. M. \& Orfeo, T. One Hundred and Twenty-Seven Cultured Human Tumor Cell Lines Producing Tumors in Nude Mice. JNCI J. Natl. Cancer Inst. 59, 221226 (1977).

20. Shin, G. W. et al. Targeted short read sequencing and assembly of re-arrangements and candidate gene loci provide megabase diplotypes. Nucleic Acids Res. 47, e115 (2019).

21. Tate, J. G. et al. COSMIC: the Catalogue Of Somatic Mutations In Cancer. Nucleic Acids Res. 47, 941-947 (2018).

22. Bamford, S. et al. The COSMIC (Catalogue of Somatic Mutations in Cancer) database and website. Br. J. Cancer 91, 355-358 (2004).

23. Nattestad, M. et al. Complex rearrangements and oncogene amplifications revealed by long-read DNA and RNA sequencing of a breast cancer cell line 2. (2018). doi:10.1101/174938

24. Neve, R. M. et al. A collection of breast cancer cell lines for the study of functionally distinct cancer subtypes. Cancer Cell 10, 515-527 (2006).

25. Robinson, J. T. et al. Integrative genomics viewer. Nature Biotechnology 29, 24-26 (2011).

26. Aganezov, S. et al. Comprehensive analysis of structural variants in breast cancer genomes using single molecule sequencing. bioRxiv 847855 (2019). doi:10.1101/847855

27. Jeffares, D. C. et al. Transient structural variations have strong effects on quantitative traits and reproductive isolation in fission yeast. Nat. Commun. 8, 1-11 (2017).

28. Wala, J. A. et al. SvABA: Genome-wide detection of structural variants and indels by local assembly. Genome Res. 28, 581-591 (2018).

29. Layer, R. M., Chiang, C., Quinlan, A. R. \& Hall, I. M. LUMPY: A probabilistic framework for structural variant discovery. Genome Biol. 15, R84 (2014).

30. Chen, $X$. et al. Manta: Rapid detection of structural variants and indels for germline and cancer sequencing applications. Bioinformatics 32, 1220-1222 (2016).

31. Spies, N. et al. Genome-wide reconstruction of complex structural variants using read clouds. Nat. Methods 14, 915-920 (2017).

32. Elyanow, R., Wu, H. T. \& Raphael, B. J. Identifying structural variants using linked-read sequencing data. Bioinformatics 34, 353-360 (2018).

33. Zheng, G. X. Y. et al. Haplotyping germline and cancer genomes with high-throughput linked-read sequencing. Nat. Biotechnol. 34, 303-311 (2016).

34. Farek, J. et al. xAtlas: Scalable small variant calling across heterogeneous nextgeneration sequencing experiments. bioRxiv 295071 (2018). doi:10.1101/295071

35. Luo, R. et al. Exploring the limit of using a deep neural network on pileup data for germline variant calling. Nat. Mach. Intell. 2, 220-227 (2020).

36. Miller, D. E. et al. Targeted long-read sequencing identifies missing disease-causing variation. Am. J. Hum. Genet. 108, 1436-1449 (2021).

37. Sambrook, J. \& Russell, D. W. Molecular Cloning: A Laboratory Manual. Cold Spring Harbor Laboratory Press (2001). Available at: http://www.molecularcloning.com/. (Accessed: 22nd September 2018)

38. Jain, M. et al. Nanopore sequencing and assembly of a human genome with ultra-long reads. Nat. Biotechnol. 36, 338-345 (2018).

39. Montague, T. G., Cruz, J. M., Gagnon, J. A., Church, G. M. \& Valen, E. CHOPCHOP: a CRISPR/Cas9 and TALEN web tool for genome editing. Nucleic Acids Res. 42, W401W407 (2014).

40. Labun, K., Montague, T. G., Gagnon, J. A., Thyme, S. B. \& Valen, E. CHOPCHOP v2: a web tool for the next generation of CRISPR genome engineering. Nucleic Acids Res. 44, W272-W276 (2016). 
41. Gilpatrick, T. et al. Targeted nanopore sequencing with Cas9-guided adapter ligation. Nat. Biotechnol. 38, 433-438 (2020).

42. Li, H. Minimap2: pairwise alignment for nucleotide sequences. Bioinformatics 34, 30943100 (2018).

43. Li, H. et al. The Sequence Alignment/Map format and SAMtools. Bioinformatics 25, 2078-2079 (2009).

44. Depristo, M. A. et al. A framework for variation discovery and genotyping using nextgeneration DNA sequencing data. Nat. Genet. 43, 491-501 (2011).

45. Danecek, P. et al. The variant call format and VCFtools. Bioinforma. Appl. NOTE 27, 2156-2158 (2011).

46. Quinlan, A. R. \& Hall, I. M. BEDTools: a flexible suite of utilities for comparing genomic features. Bioinforma. Appl. NOTE 26, 841-842 (2010). 